\title{
Confinement effects on decay rate of surface electron states over liquid helium
}

\author{
S.S. Sokolov ${ }^{1}$, J.M. Villas-Bôas ${ }^{2}$, Yu.P. Monarkha ${ }^{1}$, and Nelson Studart ${ }^{3}$ \\ ${ }^{1}$ B. Verkin Institute for Low Temperature Physics and Engineering of the National Academy of Sciences of Ukraine \\ 47 Lenin Ave., Kharkov 61103, Ukraine \\ E-mail: sokolov@ilt.kharkov.ua \\ ${ }^{2}$ Walter Schottky Institut, Technische Universität München, Am Coulombwall, D-85748 Garching, Germany \\ ${ }^{3}$ Departamento de Física, Universidade Federal de São Carlos, 13565-905, São Carlos, São Paulo, Brazil
}

Received November 23, 2007

\begin{abstract}
The decay rate of excited states of surface electrons in liquid helium, trapped in a quantum dot system, is evaluated, taking into account the process of spontaneous radiation of two-ripplons with short wavelength. We find that the values of the decay rate in later process are rather higher than those for the one-ripplon process previously calculated. The upper-bound limit lifetime of excited states of surface electrons in a quantum dot is found to be $\tau<10^{-6}-10^{-7} \mathrm{~s}$.
\end{abstract}

PACS: 73.20.-r Electron states at surfaces and interfaces;

73.20.-b Electron states and collective excitations in multilayers, quantum wells, mesoscopic, and nanoscale systems;

73.90.+f Other topics in electronic structure and electrical properties of surfaces, interfaces, thin films, and low-dimensional structures.

Keywords: quantum dot, two-ripplon process, lifetime of excited states.

Surface electrons (SEs) localized over the liquid helium surface have been an remarkable archetype for studying confined charged systems of low dimensionality $[1,2]$. In particular and more important, SE systems have been intensively investigated in the last years as potential candidate of qubit generators in quantum computation $[3,4]$. The main reasons for this are twofold. Firstly, the energy spectrum of SEs is quite simple and well-known. In the limit of zero holding electric field $E_{\perp}$ oriented in the normal direction to the electron layer, the spectrum is given by hydrogenic-like subbands

$$
\Delta_{l}=-\frac{m \Lambda_{0}^{2}}{2 \hbar^{2} l^{2}} ; \quad l=1,2,3 \ldots,
$$

where $\Lambda_{0}=\left(e^{2} / 4\right)\left(\varepsilon_{\mathrm{He}}-1\right) /\left(\varepsilon_{\mathrm{He}}+1\right), \varepsilon_{\mathrm{He}} \simeq 1.0572$ is liquid helium dielectric constant, $e$ and $m$ are electron charge and mass, respectively. The total electron energy is given by $E_{\mathbf{k}, l}=E_{\mathbf{k}}+\Delta_{l} ; E_{\mathbf{k}}=\hbar^{2} k^{2} /(2 m)$ where $\mathbf{k}$ is $2 \mathrm{D}$ electron wave vector in the helium liquid-vapor interface. For nonzero holding field, the dependence $\Delta_{l}\left(E_{\perp}\right)$ can be calculated within the variational approach
$[5,6]$ (see Fig. 1 for $l=1$ and 2). The minimum energy gap between ground $l=1$ and first excited $(l=2)$ subbands is $\Delta_{21}=\Delta_{2}-\Delta_{1} \simeq 5.7 \mathrm{~K}$ for $E_{\perp}=0$ and it is increasing

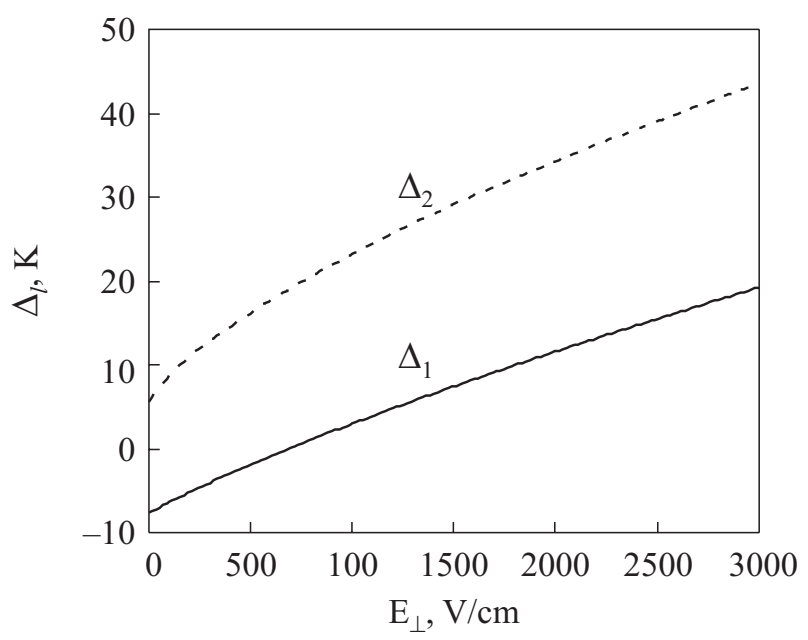

Fig. 1. The energies $\Delta_{1}$ of the ground subband $l=1$ and $\Delta_{2}$ of the first excited subband $l=2$. 
function of $E_{\perp}$, attaining, for example, 8.7 (15.5) $\mathrm{K}$ for $E_{\perp}=100(450) \mathrm{V} / \mathrm{cm}$.

Secondly, the SE scattering along the liquid-vapor interface is limited for temperatures $T<1 \mathrm{~K}$ by ripplons, the quantized capillary waves of the He surface, whose dispersion law is $\omega_{\mathbf{q}} \simeq(\alpha / \rho) q^{3 / 2}$ ( $\alpha$ and $\rho$ are the He surface tension coefficient and bulk density, respectively, $q$ is wave number). The strength of the electron-ripplon interaction decreases strongly with $T$ which favours the experimental study of qubits produced by SE. One should emphasize that even though the electron-ripplon interaction is essential to define both transport phenomena [2] and decay rate of excited electron states it is rather weak to influence the subband structure. The problem of the decay rate and the subband lifetime $\tau$, which is inversely proportional to decay rate, was first discussed in Ref. 4. The authors concluded that the contribution of the oneripplon scattering processes predominates. In such a case the SE scattering is due to long wavelength ripplons $\left(q \leq 10^{6}-10^{7} \mathrm{~cm}^{-1}\right.$ ) and $\tau$ is inversely proportional to temperature attaining rather optimistic values $\tau \sim 10^{-5}-10^{-4} \mathrm{~s}$ for $T$ in the range of millikelvin. Furthermore a strong increase of the lifetime due to confinement effects in the plane of electron layer was predicted in presence of magnetic field normal to SE system.

However, the situation is quite different if one takes into account the contribution of SE scattering by short wavelength ripplons [7]. This process occurs because the nonlinearity of the electron-ripplon interaction. As it was shown in Ref. 7 even at very low temperature the SE can radiate a pair of short wavelength ripplons in almost opposite directions. The total energy of the ripplons $2 \hbar \omega_{\mathbf{q}}$ can be of order of $E_{\mathbf{k}, l}$ and the ripplon-pair momentum satisfies the condition $s=\left|\mathbf{q}+\mathbf{q}^{\prime}\right|<<q$. The two-ripplons process is responsible for the energy relaxation of SE system [7]. It was shown that its contribution to the decay rate of the excited level $l=2$ is two orders of magnitude higher than that of one-ripplon process, i.e., $\tau \sim 10^{-6}$ $10^{-7} \mathrm{~s}$ [8]. Furthermore confinement effects due to the presence of a perpendicular magnetic field, which now makes discrete the energy $E_{\mathbf{k}}$ (Landau spectrum), do not alter substantially the values of $\tau$.
Recently, the trap of SE in a quantum dot with few electrons by specially designed electrostatic gates was realized [9]. Besides the fact that magnetic and electrostatic confinements have different physical and practical implications, the probability of SE transitions between in-plane energy levels in quantum dot do not depend on the SE momentum which leads to different matrix elements for the interaction potential. Accordingly the calculation of the SE lifetime and decay rate in quantum dot is required. The aim of present work is to perform the theoretical calculation of the decay rate in quantum dot due to the two-ripplon creation process.

The discrete energy spectrum of the SE in a quantum dot, in the asymmetrical parabolic approximation with characteristic frequencies $\omega_{x}$ and $\omega_{y}$, is written as

$$
E_{n_{x}, n_{y}, l}=\hbar \omega_{x}\left(n_{x}+\frac{1}{2}\right)+\hbar \omega_{y}\left(n_{y}+\frac{1}{2}\right)+\Delta_{l},
$$

where $n_{x}, n_{y}=0,1,2 \ldots$ Here we assume the axis $z$ to be normal to the electron layer located in the $x y$ plane. In this approximation, the wave functions for the motion along $x$ and $y$ are given in terms of Hermite functions.

The decay rate and lifetime for both excited level $l=2$ and fundamental level $l=1$ is evaluated by considering the in-plane excited states with $n_{x}, n_{y} \geq 1$. Note that the decay rate defines the linewidth of the spectroscopic transitions between the SE energy levels [10].

We start from the basic relation for energy state decay rate defined by the interaction given by the Hamiltonian $\hat{H}(\mathbf{r}, z, t)[11]$

$$
\Gamma_{\{j\}}=\frac{1}{\hbar^{2}} \sum_{\left\{j^{\prime}\right\}} \int_{-\infty}^{\infty} d t \exp \left[i \frac{\left(E_{\left\{j^{\prime}\right\}}-E_{j}\right) t}{\hbar}\right]\left\langle U_{\left\{j j^{\prime}\right\}}(0) U_{\left\{j j^{\prime}\right\}}(t)\right\rangle
$$

where $U_{\left\{j j^{\prime}\right\}}(t)=\left\langle\left\{j^{\prime}\right\}|\hat{H}(\mathbf{r}, z, t)|\{j\}\right\rangle$ is the ensemble-averaged matrix element for the states $\left\{j^{\prime}\right\}$ and $\{j\}, \mathbf{r}=\{x, y\}$ is $2 \mathrm{D}$ position-vector in the plane of the electron layer. A straightforward calculation leads to the following expression for the decay rate in a quantum dot due to the tworipplon process:

$$
\begin{aligned}
\Gamma_{\left\{n_{x}, n_{y}, l\right\}}^{(2 r)} \simeq \frac{4 \pi}{\hbar} \sum_{n_{x}^{\prime}, n_{y}^{\prime}, l^{\prime}} \sum_{\mathbf{q}, \mathbf{s}} Q_{\mathbf{q}}^{2} Q_{\mathbf{q}-\mathbf{s}}^{2}\left|\left\langle l^{\prime}\left|V_{\mathbf{q}}^{(2 r)}\right| l\right\rangle\right|^{2}\left|J_{n_{x}, n_{x}^{\prime}}\left(s_{x}\right)\right|^{2}\left|J_{n_{y}, n_{y}^{\prime}}\left(s_{y}\right)\right|^{2}\left(N_{\mathbf{q}}+1\right)\left(N_{\mathbf{q}-\mathbf{s}}+1\right) \times \\
\quad \times \delta\left[\Delta_{l^{\prime}}-\Delta_{l}+\hbar \omega_{x}\left(n_{x}^{\prime}-n_{x}\right)+\hbar \omega_{y}\left(n_{y}^{\prime}-n_{y}\right)+\hbar \omega_{\mathbf{q}}+\hbar \omega_{\mathbf{q}-\mathbf{s}}\right] .
\end{aligned}
$$

Here only radiation processes are included. In Eq. (4) and, $N_{\mathbf{q}}(T)$ is the ripplon thermal distribution function $Q_{\mathbf{q}}^{2}=\hbar q /\left(2 \rho \omega_{\mathbf{q}}\right)$. The matrix elements for in-plane electron motion are given by

$$
\left|J_{n_{x}, n_{x}^{\prime}}\left(s_{j}\right)\right|^{2}=\frac{\left[\min \left(n_{j}, n_{j}^{\prime}\right)\right] !}{\left[\max \left(n_{j}, n_{j}^{\prime}\right)\right] !}\left(\frac{s_{j}^{2} \lambda_{j}^{2}}{2}\right)^{\left|n_{j}-n_{j}^{\prime}\right|} \exp \left(-\frac{s_{j}^{2} \lambda_{j}^{2}}{2}\right)\left[L \underset{\min \left(n_{j}, n_{j^{\prime}}\right)}{\left|n_{j}-n_{j}^{\prime}\right|}\left(\frac{s_{j}^{2} \lambda_{j}^{2}}{2}\right)\right]^{2},
$$


where $L_{v}^{\beta}(x)$ are Laguerre polynomials and $\lambda_{j}^{2}=\hbar /\left(m \omega_{j}\right)$ with $\omega_{j}=\omega_{x}$ if $j=x$ and $\omega_{j}=\omega_{y}$ if $j=y$. The structure of the matrix elements $\left\langle l^{\prime}\left|V_{\mathbf{q}}^{(2 r)}\right| l\right\rangle$ for the two-ripplon process is rather interesting. As it was shown in Ref. 2 (see also Ref. 8) the matrix elements for the two-ripplon process in the electron-ripplon scattering should be calculated within the Bloch treatment for ripplon wave numbers exceeding $q \simeq 2 \cdot 10^{7} \mathrm{~cm}^{-1}$. For such $q^{6}$ s, typical for two-ripplon processes which are quadratic in the displacement $\xi(\mathbf{r})$ of the liquid-vapor interface from the equilibrium position $z=0$, one has

$$
\left\langle l^{\prime}\left|V_{\mathbf{q}}^{(2 r)}\right| l\right\rangle=\kappa_{0} \sqrt{\left(\frac{\partial v}{\partial z}\right)_{l l}\left(\frac{\partial v}{\partial z}\right)_{l^{\prime} l^{\prime}}},
$$

where $(\partial v / \partial z)_{l l}=\langle l|v(z)| l\rangle, v(z)=-\Lambda_{0} / z+e E_{\perp} z$ and $\kappa_{0}=\sqrt{2 m V_{0}} / \hbar$. Indeed the matrix elements given by Eq. (6) depend explicitly on the height of the potential barrier $V_{0} \simeq 1 \mathrm{eV}$ which prevents SE penetrating inside the liquid helium. The wave functions $f_{l}(z)$ for $l=1$ and $l=2$ are calculated within the variational approach $[5,6]$.

Taking into account that $N_{\mathbf{q}}<<1$ and disregarding $s$ in comparison with $q$ in Eq. (4), we arrive to the following expressions for the decay rates for $l=2$ and $l=1$ :

$$
\begin{aligned}
& \Gamma_{\left(n_{x}, n_{y}, 2\right)}^{(2 r)}=\frac{\kappa_{0}^{2}}{12 \pi^{2} \alpha^{3 / 2} \rho^{1 / 2} \lambda_{x} \lambda_{y}} \times \\
& \times \sum_{n_{x}^{\prime} n_{y}^{\prime}} I_{n_{x} n_{x}^{\prime}} I_{n_{y} n_{y}^{\prime}}\left[\frac{\left(\frac{\partial \mathrm{v}}{\partial z}\right)_{22}^{2}}{q_{0}^{1 / 2}}+\frac{\left(\frac{\partial \mathrm{v}}{\partial z}\right)_{11}\left(\frac{\partial \mathrm{v}}{\partial z}\right)_{22}}{\left(q_{0}^{*}\right)^{1 / 2}}\right] \\
& \Gamma_{\left(n_{x}, n_{y}, 1\right)}^{(2 r)}=\frac{\kappa_{0}^{2}}{12 \pi^{2} \alpha^{3 / 2} \rho^{1 / 2} \lambda_{x} \lambda_{y}} \times \\
& \times \sum_{n_{x}^{\prime} n_{y}^{\prime}} I_{n_{x} n_{x}^{\prime}} I_{n_{y} n_{y}^{\prime}}\left[\frac{\left(\frac{\partial v}{\partial z}\right)_{11}^{2}}{q_{0}^{1 / 2}}+\frac{\left(\frac{\partial v}{\partial z}\right)_{11}\left(\frac{\partial v}{\partial z}\right)_{22}}{\left(q_{0}^{* *}\right)^{1 / 2}}\right] \text {, }
\end{aligned}
$$

where the characteristic ripplon wave numbers are

$$
\begin{gathered}
q_{0}=(\rho / 4 \alpha)^{1 / 3}\left[\omega_{x}\left(n_{x}-n_{x}^{\prime}\right)+\omega_{y}\left(n_{y}-n_{y}^{\prime}\right)\right]^{2 / 3} ; \\
q_{0}^{*}=(\rho / 4 \alpha)^{1 / 3}\left[\Delta_{21} / \hbar+\omega_{x}\left(n_{x}-n_{x}^{\prime}\right)+\omega_{y}\left(n_{y}-n_{y}^{\prime}\right)\right]^{2 / 3} ; \\
q_{0}^{* *}=(\rho / 4 \alpha)^{1 / 3}\left[-\Delta_{21} / \hbar+\omega_{x}\left(n_{x}-n_{x}^{\prime}\right)+\omega_{y}\left(n_{y}-n_{y}^{\prime}\right)\right]^{2 / 3}
\end{gathered}
$$

and

$$
\begin{gathered}
I_{n_{j}, n_{j}^{\prime}}=\frac{\left[\min \left(n_{j}, n_{j}^{\prime}\right)\right] !}{\left[\max \left(n_{j}, n_{j}^{\prime}\right)\right] !} \times \\
\times \int_{0}^{\infty} x^{\left|n_{j}-n_{j}^{\prime}\right|-1 / 2} \exp (-x)\left[L_{\min \left(n_{j}, n_{j}^{\prime}\right)}^{\left|n_{j}-n_{j}^{\prime}\right|}(x)\right]^{2} d x .
\end{gathered}
$$

The sums which appear in Eqs. (7) and (8) are carried out over the values of $n_{x}^{\prime}$ and $n_{y}^{\prime}$ satisfying the condition of positiveness of the expressions in square brackets of $q_{0}$, $q_{0}^{*}$, and $q_{0}^{* *}$, respectively. One emphasizes that the decay rate does not depend on temperature which is a direct consequence of neglecting $N_{\mathbf{q}}(T)$ in Eq. (4) because it is very small for short wavelength ripplons. On the contrary, for long wavelength ripplons $N_{\mathbf{q}} \simeq T / \hbar \omega_{\mathbf{q}}>1$. Therefore a linear $T$-dependence of the decay rate is obtained when only the one-ripplon process is considered [4].

The first term in the sums of Eqs. (7) and (8) correspond to intrasubband scattering contributions whereas the second term is related to intersubband scattering.

Looking over the expressions for the decay rates one must emphasize that the matrix elements given by Eq. (5) depend on $s_{j}^{2} \lambda_{j}^{2} / 2 \sim 1$ for $s<<q$. Likewise the calculation for the one-ripplon process leads to the matrix elements which depend on $q_{j} \lambda_{j} \gg 1$. Consequently the matrix elements in Eq. (5) being proportional to $\exp \left(-q_{j}^{2} \lambda_{j}^{2} / 2\right)<<1$ are quite small. The conclusion is that the contribution from one-ripplon process for the decay rate of SE in a quantum dot is absolutely negligible in comparison with that from two-ripplon process.

In Fig. 2 we depicted the dependence of the lifetimes $\tau_{2}^{(2 r)}=\left[\Gamma_{\left\{n_{x}, n_{y}, 2\right\}}^{(2 r)}\right]^{-1}$ and $\tau_{1}^{(2 r)}=\left[\Gamma_{\left\{n_{x}, n_{y}, 1\right\}}^{(2 r)}\right]^{-1}$ on the holding electric field for $n_{x}=1$ and $n_{y}=0$. The energies of the parabolic confinement are $\hbar \omega_{x}=0.5 \mathrm{~K}$ and $\hbar \omega_{y}=0.6 \mathrm{~K}$, respectively. We see that the order of magnitude of the

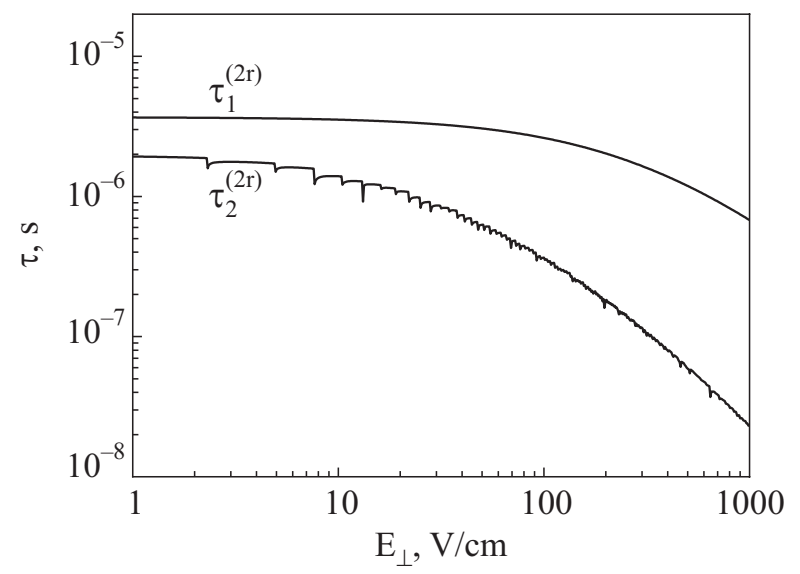

Fig. 2. The lifetime $\tau_{2}^{(2 r)}$ for the SE excited states $\left\{n_{x}=1\right.$; $\left.n_{y}=0, l=2\right\}$ and $\tau_{1}^{(2 r)}$ for $\left\{n_{x}=1 ; n_{y}=0 ; l=1\right\}$ limited by the two-ripplon scattering process. 
lifetimes is $10^{-6} \mathrm{~s}$ for $E_{\perp}=0$ and both $\tau_{2}^{(2 r)}$ and $\tau_{1}^{(2 r)}$ decrease strongly as the holding electric field increases. Note that the values of $\tau_{2}^{(2 r)}$ are close to those obtained in Ref. 8 both for free electron motion in $x y$ plane and in presence of magnetic field along $z$. We also observe the values of $\tau_{1}^{(2 r)}$ are higher than those of $\tau_{2}^{(2 r)}$. We should remark that only the first term of the intrasubband scattering in the sum of Eq. (8) contributes to $\Gamma_{\left\{n_{x}, n_{y}, 1\right\}}^{(2 r)}$ and consequently to $\tau_{1}^{(2 r)}$ for the chosen $\hbar \omega_{x}=0.5 \mathrm{~K}$ and $\hbar \omega_{y}=0.6 \mathrm{~K}$. The sharp difference between the values of $\tau_{2}^{(2 r)}$ and $\tau_{1}^{(2 r)}$ may be favourable in measurements of the spectroscopic linewidth in a quantum dot. Indeed the signals of the transitions from the excited SE subband $l=2$ and from excited states of in-plane motion with $n_{x}, n_{y} \geq 1$ for $l=1$ should be clearly distinguished. Interestingly enough are the oscillations appearing in $\tau_{2}^{(2 r)}\left(E_{\perp}\right)$ whose origin comes from the denominator of the second term of the sum in Eq. (7). One can easily check that there is a square root singularity for $\Delta_{21} / \hbar$ close to $\omega_{x}\left(n_{x}^{\prime}-n_{x}\right)+$ $+\omega_{y}\left(n_{y}^{\prime}-n_{y}\right)$. For example for $E_{\perp}=2.3 \mathrm{~V} / \mathrm{cm}\left(\Delta_{21} \simeq\right.$ $\simeq 5.8 \mathrm{~K})$ the $q_{0}^{*}$ trends to zero for $\left\{n^{\prime}{ }_{x}=3 ; n^{\prime}{ }_{y}=8\right\}$ and $\left\{n_{x}^{\prime}=9 ; n_{y}^{\prime}=3\right\}$. Similar oscillations were observed for $\mathrm{SE}$ decay rate under magnetic field [8]. One should stress that the Eq. (4), obtained for the process of creation of two short wavelength ripplons with $q>10^{7} \mathrm{~cm}^{-1}$, becomes invalid for $q \rightarrow 0$. For this reason the neighborhood of this singularity should be treated appropriately by taking into account on equal footing both two-ripplons and one-ripplon processes. On the other hand, note that the first term in the sum of Eq. (8) has no singularities. In this case, only the state $n^{\prime}{ }_{x}=n^{\prime}{ }_{y}=0$ does contribute to $\Gamma_{\left(n_{x}, n_{y}, 1\right)}^{(2 r)}$ for all holding fields. As a result, a smooth dependence $\tau_{1}^{(2 r)}\left(E_{\perp}\right)$ is obtained.

In conclusion we determined the decay rate of excited states of surface electrons on liquid helium trapped in a quantum dot. We showed that both decay rate and lifetime of the excited states do not depend on temperature and are limited by the process of spontaneous creation of a pair of short wavelength ripplons (with wave numbers exceeding $10^{7} \mathrm{~cm}^{-1}$ ). The values of the decay rate are essentially higher and therefore the lifetimes are much smaller than those limited by one-ripplon process [4]. The results obtained here should be essential for the experimental study of qubits based on surface electrons on helium.

The work was partially supported by FAPESP, CNPq, and by STCU Project 3718. S.S.S thanks the hospitality of the Physics Department of the Federal University of São Carlos where part of this work was developed. J.M.V.B is supported by a Humboldt Foundation fellowship.

1. Two-Dimensional Electron Systems on Helium and Other Cryogenic Substrates, E.Y. Andrei (ed.), Kluwer Academic, Dordrecht (1997).

2. Yu.P. Monarkha and K. Kono, Two-Dimensional Coulomb Liquids and Solids, Springer, Berlin (2004).

3. P.M. Platzman and M.I. Dykman, Science 284, 1967 (1999); M.J. Lea, P.G. Frayne, and Yu. Mukharsky, Fortschr. Phys. 48, 1109 (2000); Arnold J. Dahm, J.A. Heilman, I. Karakurt, and T.J. Peshek, Physica E18, 169 (2003).

4. M.I. Dykman, P.M. Platzman, and P. Seddighrad, Phys. Rev. B67, 155402 (2003).

5. Yu.P. Monarkha, S.S. Sokolov, and V.B. Shikin, Solid State Commun. 38, 611 (1981).

6. S.S. Sokolov, Fiz. Nizk. Temp. 30, 271 (2004) [Low Temp. Phys. 30, 199 (2004)].

7. Yu.P. Monarkha, Fiz. Nizk. Temp. 4, 1093 (1978) [Sov. J. Low Temp. Phys. 4, 515 (1978)].

8. Yu.P. Monarkha and S.S. Sokolov, J. Low Temp. Phys. 148, 157 (2007).

9. E. Rousseau, Y. Mukharsky, D. Ponarine, O. Avenel, and E. Varoquaux, J. Low Temp. Phys. 148, 193 (2007).

10. T. Ando, J. Phys. Soc. Jpn. 44, 765 (1978).

11. V.B. Shikin and Yu.P. Monarkha, J. Low Temp. Phys. 16, 193 (1974). 